\title{
Reaktive neutrophile Dermatosen
}

\section{Reactive Neutrophilic Dermatosis}

Autoren

Institute
E. Kühl' ${ }^{1}$ M. Wolter ${ }^{2}$, C. Löser ${ }^{1}$, E. Dippel ${ }^{1}$

1 Hautklinik, Klinikum der Stadt Ludwigshafen, Ludwigshafen

2 Klinik für Dermatologie, Venerologie und Allergologie, Johann Wolfgang Goethe-Universität Frankfurt am Main

\section{Bibliografie}

DOI http://dx.doi.org/

10.1055/s-0029-1244057

Online-Publikation: 24. 3. 2010

Akt Dermatol 2010; 36:

165-170 @ Georg Thieme

Verlag KG Stuttgart · New York

ISSN 0340-2541

\section{Korrespondenzadresse}

Prof. Dr. Edgar Dippel

Hautklinik

Klinikum der Stadt Ludwigshafen $\mathrm{GmbH}$

Bremserstr. 79

67063 Ludwigshafen

dippele@klilu.de

\section{Zusammenfassung \\ $\nabla$}

Reaktive neutrophile Dermatosen wie das Pyoderma gangraenosum und die akute febrile neutrophile Dermatose (Sweet-Syndrom) zeigen bei unterschiedlichen klinischen Erscheinungsbildern ähnliche pathologische Merkmale der Entzündung am Hautorgan. Histopathologisch finden sich Infiltrate von normalen polymorphkernigen Leukozyten, eine erhöhte Anzahl von Granulozyten im peripheren Blut und die häufige Assoziation mit systemischen Erkrankungen. Am

\section{Einleitung}

Nahezu bei allen rheumatologischen Erkrankungen kann es zu einer reaktiven Beteiligung des Hautorgans kommen. Häufig ist die Hautbeteiligung, insbesondere bei Kollagenosen oder Vaskulitiden, ein diagnostisches Leitsymptom. Die Gruppe der reaktiven neutrophilen Dermatosen hat in dieser Hinsicht eine besondere Bedeutung, da die klinischen Symptome oft mit der Akuität der Grunderkrankung vergesellschaftet sind. Jedes Körperorgan, aber auch jede Struktur der Haut kann von einer aseptischen neutrophilen Entzündungsreaktion betroffen sein. Zu den reaktiven neutrophilen Dermatosen gehören das Pyoderma gangraenosum, die akute febrile neutrophile Dermatose (Sweet-Syndrom), die subkorneale Pustulose und sehr selten die rheumatoide neutrophile Dermatitis und die neutrophile ekkrine Hidradenitis ( Tab. 1) [1,2].

In einigen Fällen können sich diese Symptome auch überlappen oder gleichzeitig auftreten. Dabei ergeben sich zwei polarisierende klinische Manifestationen - zum einen die infiltrierten sukkulenten Plaques beim Sweet-Syndrom und zum anderen die ulzerierenden Läsionen beim Pyoderma gangraenosum. Die Pathogenese beider Erkrankungen ist noch unklar. Doch gibt es Hin- häufigsten handelt es sich dabei um chronische gastrointestinale, rheumatologische oder hämatologische Erkrankungen, die sich zum Zeitpunkt der Hauterscheinungen in einer akuten Phase oder Krankheitverschlechterung befinden. Die genauen pathopysiologischen Bedingungen zur Ausprägung einer reaktiven neutrophilen Dermatose sind nicht bekannt, jedoch ist die Therapie mit systemischen antientzündlichen Medikamenten und insbesondere durch die anti-TNF $\alpha$ Antikörper-Behandlung in den meisten Fällen effektiv.

weise, dass die immunologische Reaktivität oder Pathergie durch Zytokine wie IL-1, IL-3, IL-6, IL-8, G-CSF und TNF $\alpha$ beeinflusst werden $[3,4]$. Die häufige Assoziation mit entzündlichen Systemerkrankungen und das gute Ansprechen auf antientzündliche oder immunmodulierende Therapien sind weitere indirekte pathogenetische Hinweise. Im Folgenden berichten wir über reaktive neutrophile Dermatosen und demonstrieren klinische Verläufe von Patienten mit Pyoderma gangraenosum, Sweet-Syndrom und der sehr seltenen abszedierenden neutrophilen Dermatose.

\section{Fallberichte \\ $\nabla$}

\section{Fall 1}

Anamnese. Die 18-jährige Patientin wurde zunächst in einer chirurgischen Klinik stationär aufgenommen zur Abklärung blutiger Stühle, die seit einigen Wochen bestanden. Innerhalb weniger Tage kam es zum Auftreten von Pusteln am gesamten Integument. Daraufhin erfolgte die Verlegung der Patientin in die Hautklinik. Bei Aufnahme befand sich die Patientin in einem deutlich reduzierten Allgemeinzustand.

Befunde. Hautbefund: Am gesamten Integument fanden sich disseminiert Pusteln einschließlich 
Tab. 1 Spektrum der neutrophilen Dermatosen in Assoziation mit sytemischen Erkankungen.

\begin{tabular}{|c|c|}
\hline & Assoziierte Erkrankungen und \\
\hline Dermatosen & Ursachen \\
\hline Pyoderma gangraenosum & $\begin{array}{l}\text { Leukämien, Myelome (Paraprotein- } \\
\text { ämie), Colitis ulcerosa, M. Crohn, } \\
\text { M. Behçet, HIV, Sarkoidose, chronisch } \\
\text { aktive Hepatitis, GCSF-Gabe, Medika- } \\
\text { mente }\end{array}$ \\
\hline $\begin{array}{l}\text { Akute neutrophile Dermatose } \\
\text { (Sweet's Syndrome) }\end{array}$ & $\begin{array}{l}\text { Colitis ulcerosa, M. Crohn, Leukämien, } \\
\text { Myeloproliferative Erkrankungen, } \\
\text { Karzinome, Bindegewebserkran- } \\
\text { kungen, Schilddrüsenerkrankungen, } \\
\text { Rheumatoide Arthritis, Infektionen, } \\
\text { GCSF-Gabe }\end{array}$ \\
\hline Subkorneale Pustulosis & $\begin{array}{l}\text { IgA-Gammopathie, Colitis ulcerosa, } \\
\text { M. Crohn, Rheumatoide Arthritis, } \\
\text { Myeloproliferative Erkrankungen, } \\
\text { solide Tumore, Medikamente }\end{array}$ \\
\hline $\begin{array}{l}\text { Neutrophile ekkrine } \\
\text { Hidradenitis }\end{array}$ & $\begin{array}{l}\text { Leukämien +/- Chemotherapie, } \\
\text { solide Tumore, M. Behçet, Infek- } \\
\text { tionen, Medikamente }\end{array}$ \\
\hline $\begin{array}{l}\text { Rheumatoide neutrophile } \\
\text { Dermatose }\end{array}$ & Rheumatoide Arthrits \\
\hline $\begin{array}{l}\text { Abzedierende neutrophile } \\
\text { Dermatose }\end{array}$ & $\begin{array}{l}\text { Myelodysplasitisches Syndrom, IgA- } \\
\text { Myelom, M. Crohn, Paraproteinämie }\end{array}$ \\
\hline
\end{tabular}

des Capillitiums sowie prominent retroaurikulär rechts, im Bereich der Gingiva und perianal, welche sich im Verlauf teilweise ulzerierten und ausdehnten.

Koloskopie: Bereits im proximalen Sigma erfolgte ein Abbruch der Untersuchung bei ausgeprägter Entzündung und schwerer ulzeröser Kolitis mit teils sehr tiefen Ulzera. In der Histologie der Kolonschleimhautbiopsien zeigte sich in erster Linie das Bild einer Colitis ulcerosa.

Histopathologie. An der Oberfläche zeigte sich eine leicht vorgewölbte, noch intakte, zentral blasig vorgewölbte Epidermis mit reaktiver Akanthose und leichter Hyperkeratose, darunter intradermal eine abszedierende Läsion mit massenhafter Anschoppung von neutrophilen Granulozyten, wenigen Lymphozyten und Plasmazellen. Weiterhin zeigten sich ein Ödem der Haut mit Hyperämie und prominenten Papillen sowie Reste von zu Grunde gegangenen follikulären Strukturen und im Randbereich wiederholt Gefäße mit fibrinoider Wandnekrose.

Weitere Befunde: In der mikrobiologischen Diagnostik konnten keine pathogenen Keime nachgewiesen werden. Auffällige Laborparameter waren unter anderem eine CRP-Erhöhung von $18,4 \mathrm{mg} / \mathrm{dl}$, eine Leukozytose von 13,2/nl sowie ein erniedrigtes Hämoglobin von 9,0 g/dl.

Diagnose. Pyoderma gangraenosum, pustulöser Typ.

Therapie und Verlauf. Wir leiteten zunächst eine systemische Antibiose ein, zusätzlich erfolgte eine systemische Steroidtherapie mit initial Solu-Decortin H 100 mg i.v. Lokal behandelten wir mit antiseptischen Externa, eine größere abszedierte Hautveränderung retroaurikulär rechts wurde mittels Inzision entlastet und mit Polividon-Iod-Lösung gespült.

Nach vier Tagen erfolgte die Verlegung in die Gastroenterologie im Hause. Dort wurde aufgrund einer Fistel mit Abszedierung eine Omphalektomie durchgeführt. Im weiteren Verlauf wurde eine Therapie mit Cyclosporin A begonnen, hierunter waren die pustulösen und abzedierenden Hautveränderungen deutlich rückläufig.

\section{Fall 2}

Anamnese. Die 40-jährige Patientin befand sich initial in einer internistischen Klinik zur Abklärung von Hämoptypsen und trockenem Husten, bestehend seit zwei Monaten. Bronchoskopisch wurden massive granulomatöse Veränderungen des gesamten Tracheo-Bronchialsystems gesehen. Von klinischer Seite war eine Sarkoidose differenzialdiagnostisch am wahrscheinlichsten, obwohl die histologische Sicherung von entnommenen Proben keinen richtungsweisenden Befund ergab. Eine offene Tuberkulose wurde ausgeschlossen. Eine hochdosierte Steroidtherapie wurde begonnen, nach einigen Wochen schrittweise reduziert bis auf eine Dosis von $40 \mathrm{mg}$ täglich. Zeitgleich traten am Unterschenkel und an der rechten Flanke schmerzhafte knotige Hautveränderungen auf, welche rasch ulzerierten und sich vergrößerten. Anamnestisch wurden vor 20 Jahren eosinophile Granulome im Bereich beider Oberschenkel sowie des linken Fußes mit Radiatio behandelt.

Befunde. Hautbefund: Am rechten Oberschenkel ventral sah man ein ca. $4 \times 4 \mathrm{~cm}$ großes Ulkus mit livid-rötlichem Randsaum, am linken Oberschenkel ventral fand sich ein ca. $2 \mathrm{~cm}$ großes Ulkus. Am linken Unterschenkel zeigte sich ein ca. $5 \times 5 \mathrm{~cm}$ großes, livid rotes Ulkus. Am Rücken zeigte sich ein ca. $5 \times 3 \mathrm{~cm}$ großes Ulkus mit Randwall.

Histopathologie: In der dermatohistopathologischen Untersuchung zeigte sich eine mit neutrophilen Granulozyten durchsetzte Epidermis, stellenweise mit Parakeratose. Es fand sich ein kaum beteiligter subepidermaler Kollagenstreifen, unterlagernd ein massives Entzündungszellinfiltrat, bestehend überwiegend aus intakten neutrophilen Granulozyten.

Weitere Befunde: In der mikrobiologischen Diagnostik konnten keine pathogenen Keime nachgewiesen werden. Bei den Laborparametern zeigte sich eine Leukozytose von 23,4/nl, ein erniedrigtes Hämoglobin von $11,0 \mathrm{mg} / \mathrm{dl}$, eine Thrombozytose von $562 /$ nl sowie eine Neutrophilie von $84,8 \%$. Das CRP war mit $13,8 \mathrm{mg} / \mathrm{dl}$ erhöht.

Diagnose. Pyoderma gangraenosum, ulzerierter Typ.

Therapie und Verlauf. Wir leiteten zunächst eine immunsuppressive Therapie ein mit 250 mg Solu-Decortin H i.v. für zwei Tage, danach mit 100 mg täglich in absteigender Dosierung in Kombination mit Cyclosporin A mit $5 \mathrm{mg} / \mathrm{kg} / \mathrm{KG}$. Bei zögerlichem Ansprechen und Befundprogredienz entschieden wir uns für eine zusätzliche einmalige Antikörper-Gabe mit Infliximab 200 mg. Lokal kam initial Polyhexanid-Creme, zuletzt Tacrolimus 0,1\%-Salbe zum Einsatz. Darunter konnte eine rasche Abheilungstendenz der Ulzera beobachtet werden, sodass die Steroiddosis bei Entlassung auf Decortin H 20 mg täglich reduziert werden konnte ( $\bullet$ Abb. 1).

Zusätzlich erfolgte eine Reduktion der Dosierung von Cyclosporin auf $200 \mathrm{mg}$ täglich bei einem Talspiegel von $150 \mathrm{ng} / \mathrm{ml}$.

Die Schmerztherapie wurde regelmäßig optimiert. Vor der Entlassung konnte die Einnahme von Hydromorphonhydrochlorid $8 \mathrm{mg} / \mathrm{d}$ abgesetzt werden. Den steroidinduzierten Diabetes behandelten wir zuletzt mit Humaninsulin 8-5-0 IE tgl. Bezüglich der radiologisch nachgewiesenen Sarkoidose erfolgte ein Verlaufs-Thorax-CT, welches keinen Hinweis für vergrößerte Lymphknoten ergab.

\section{Fall 3}

Anamnese und Lokalbefund. Bei einem 70-jährigen Patienten zeigte sich nach einem bullösen Erysipel am Unterschenkel links ein chronisches Ulkus, sodass zunächst eine Mesh-Graft-Transplantation vom Oberschenkel links erfolgte. Im Verlauf befand 


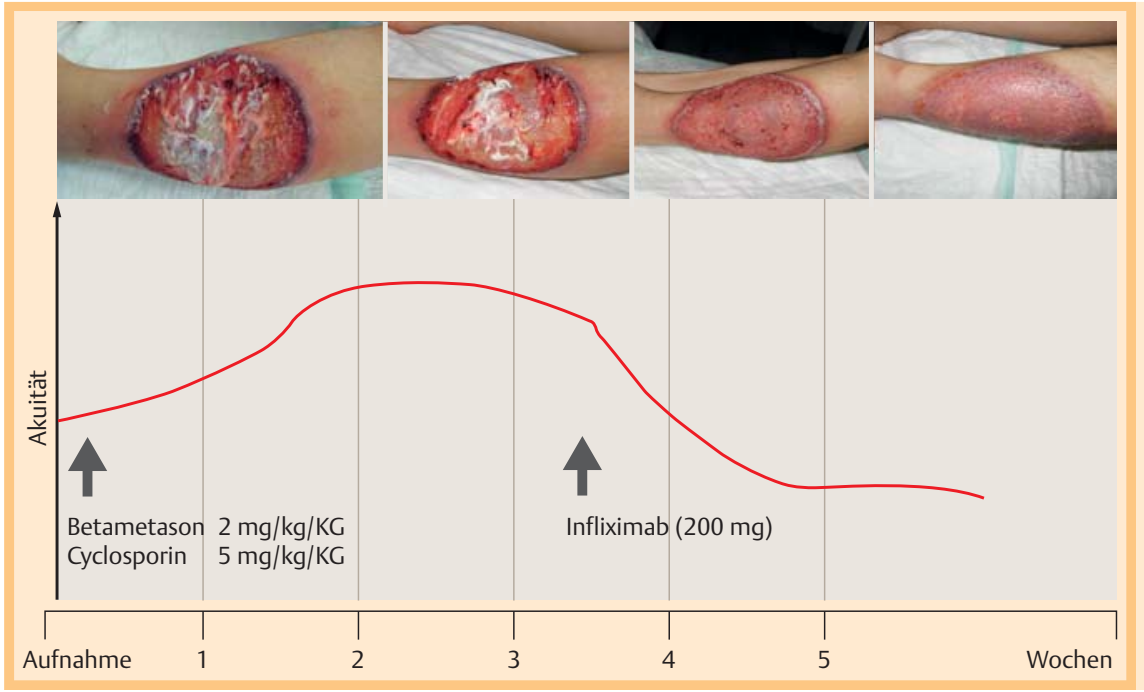

Abb. 1 Klinischer Verlauf eines ulzerienden Pyoderma gangraenosum im Bereich des Oberschenkels nach Gabe von Infliximab (Pat. 2).

sich der Patient innerhalb von 18 Monaten sechsmalig in unserer stationären Behandlung zur konservativen Therapie unter der Diagnose eines Ulcus cruris. Zuletzt hatte das Ulkus am Unterschenkel links eine Größenausdehnung von ca. $30 \times 15 \mathrm{~cm}$ erreicht. Aufgrund der Therapieresistenz wurde eine Probeexzision entnommen und eine immunsuppressive Therapie eingeleitet.

Diagnose. Pyoderma gangraenosum, vegetierender Typ.

Therapie und Verlauf. Nach Gabe von Cyclosporin A $3 \mathrm{mg} / \mathrm{kg} / \mathrm{KG}$ und Prednisolon $20 \mathrm{mg} / \mathrm{d}$ konnte innerhalb von wenigen Wochen eine nahezu vollständige Abheilung der Ulzeration erzielt werden.

\section{Fall 4}

Anamnese und Lokalbefund. Die 35-jährige Patientin befand sich in den letzten vier Jahren mehrfach in unserer stationären Behandlung bei erstmalig vor fünf Jahren aufgetreten, zunächst münzgroßen, im Verlauf an Größe zunehmenden flachen Ulzerationen mit lividem Randsaum an den Unterschenkeln beidseits (॰ Abb. 2).

Zunächst erfolgte eine hochdosierte systemische Steroidtherapie, darunter zeigte sich jedoch ein mangelndes Ansprechen der Ulzera.

Seit 8 Jahren wird die Patientin bei Z.n. Heroin-Abusus mit Methadon substituiert. Nebenbefundlich besteht eine bekannte chronische Hepatitis $\mathrm{C}$.

Diagnose. Pyoderma gangraenosum, vegetierender Typ.

Therapie und Verlauf. Unter einer Therapie mit zuletzt Azathioprin $75 \mathrm{mg}$ und Prednisolon $40 \mathrm{mg}$ waren die Ulzera deutlich rückläufig.

\section{Fall 5}

Anamnese. Bei der 66-jährigen Patientin bestanden seit vier Jahren Ulzera an beiden Unterschenkeln, seit einem halben Jahr waren diese zunehmend größenprogredient trotz ambulanter Therapie mit Prednisolon $40 \mathrm{mg}$. Eine Probeexzision wurde von der Patientin abgelehnt. Weitere Erkrankungen: arterielle Hypertonie, Z.n. Myokardinfarkt, Z.n. Apoplex, Z.n. Mamma-Karzinom, periphere arterielle Verschlusskrankheit.

Befunde. Hautbefund: An beiden Unterschenkeln zeigten sich multiple Ulzera mit lividem Randsaum und gelblichen Belägen, Durchmesser jeweils ca. $2 \times 2$ bis $4 \times 4 \mathrm{~cm}$, tibial links $8 \times 5 \mathrm{~cm}$.

Diagnose. Pyoderma gangraenosum, vegetierender Typ.

Therapie und Verlauf. Die Therapie erfolgte mit Azathioprin $100 \mathrm{mg} / \mathrm{d}$ und Prednisolon $30 \mathrm{mg} / \mathrm{d}$ für 3 Wochen. Weiterhin

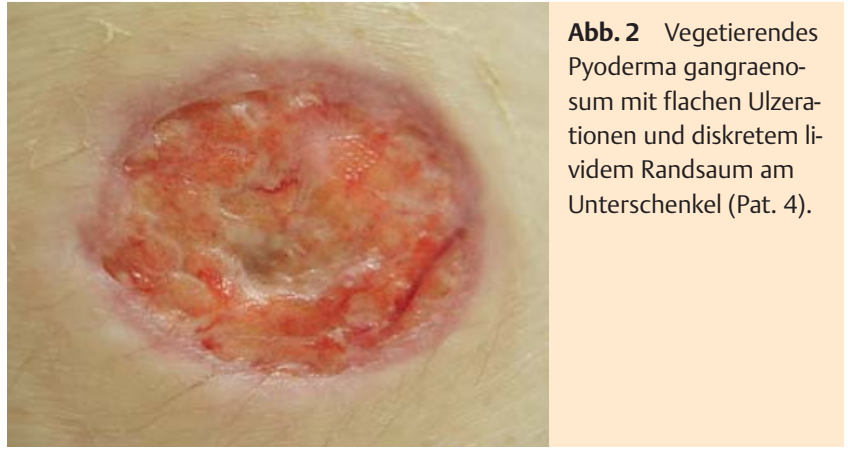

wurde eine operative Deckung mittels Meshgraft-Transplantat vom linken Oberschenkel durchgeführt. Postoperativ zeigte sich eine Anheilung des Transplantates nur am linken Unterschenkel.

\section{Fall 6}

Anamnese. Die 62-jährige Patientin berichtete bei Vorstellung über akut aufgetretene Fieberschübe bis $39,0^{\circ} \mathrm{C}$ mit begleitenden Halsschmerzen, Schluckbeschwerden und Lymphknotenschwellung nach Besuch eines Badesees. Im Verlauf traten disseminiert Erytheme an Schultern und Rücken auf, welche sich auf Arme und Beine ausbreiteten mit begleitend leichtem Juckreiz und zeitweise Schmerzhaftigkeit der Effloreszenzen. Ferner gab die Patientin Abgeschlagenheit und Nachtschweiß an.

Befunde. Hautbefund: Am oberen Rücken, an den Streckseiten der Arme beidseits und vereinzelt am Dekolleté sowie am unteren Rücken zeigten sich infiltrierte sukkulente erythematöse Plaques mit teilweise klarer Sekretion und Pseudobläschenbildung. Auch an den Oberschenkeln beidseits fanden sich einzelne erythematöse Plaques. Zervikal zeigten sich beidseits geschwollene Lymphknoten bei insgesamt deutlich reduziertem Allgemeinzustand.

Weitere Befunde: Im Rahmen des Routinelabors fielen folgende Parameter pathologisch aus: Erythrozyten 3,93/pl, Hämoglobin $11,7 \mathrm{~g} / \mathrm{dl}$, Hämatokrit 34,3\%, Thrombozyten 574/nl, Lymphozyten 22,7\%, Neutrophile 73,8\%, Eosinophile 0,2\%.

Blutsenkung > $120 \mathrm{~mm} / \mathrm{h}$. CRP $11,5 \mathrm{mg} / \mathrm{dl}$.

Diagnose. Sweet-Syndrom bei Infekt der oberen Atemwege. Therapie und Verlauf. Wir behandelten systemisch mit Steroiden, initial Prednisolon $50 \mathrm{mg}$ p.o. mit Dosisreduktion im weiteren Verlauf, sowie ergänzend mit lokalen Steroiden. Unter dieser Therapie zeigte sich rasch ein deutlicher Rückgang der Efflores- 
zenzen. Laborchemisch zeigten sich die initial erhöhten Entzündungsparameter bereits nach wenigen Tagen rückläufig.

Fall 7

Anamnese. Bei der stationären Aufnahme berichtete die 59-jährige Patientin über seit einer Woche bestehende Halsschmerzen mit Lymphknotenschwellung. Aufgrund dieser Symptomatik wurde Aspirin ${ }^{\circledR}$ über mehrere Tage eingenommen. Nach vier Tagen kam es zum Auftreten von rötlichen Plaques an den Händen mit zügiger Progression. Am Tag vor der Aufnahme zeigte sich eine Druckdolenz der Knoten mit Nekrosenbildung an der linken Hand und Bewegungseinschränkung. Weiter berichtete die Patientin über Gelenkbeschwerden in BWS, LWS und im Hüftgelenk sowie Schüttelfrost und Abgeschlagenheit.

Befunde. Hautbefund: An Gesicht und Rücken sowie an den Händen beidseits fanden sich unterschiedlich große erythematöse, indurierte und scharf begrenzte Knoten. An den Unterschenkeln beidseits sah man kleine, erythematöse Papeln. An der linken Hand fand sich zusätzlich eine etwa $2 \mathrm{~cm}$ große livide Plaque mit Bewegungseinschränkung der Hand.

Histopathologie: Unterhalb der Epidermis fand sich ein von perivaskulär auf das Interstitium übergreifendes Entzündungsinfiltrat bestehend aus stabkernigen und segmentkernigen Neutrophilen sowie Leukzytoklasie.

Weitere Befunde: Leukozyten 18,4/nl. CRP 11,8 mg/dl.

Diagnose. Sweet-Syndrom bei Infekt der oberen Atemwege DD medikamentenallergisch.

Therapie und Verlauf. Es erfolgte eine Therapie mit Prednisolon initial $100 \mathrm{mg}$ p.o. mit anschließend schrittweiser Reduktion sowie zusätzlich lokal steroidhaltigen Externa. Hierunter zeigte sich rasch ein rückläufiger Befund.

Fall 8

Anamnese. Bei der 54-jährigen Patientin waren eine Woche vor der stationären Aufnahme schmerzhafte entzündliche Hautveränderungen an den Unterschenkeln beidseits sowie am rechten Unterarm mit rascher Größenprogredienz aufgetreten, am Unterschenkel rechts auch Blasenbildung. In den vorangehenden Tagen bestanden zudem Gelenkschmerzen und Fieber.

Bereits zwei Jahre zuvor hatte sich die Patientin schon einmal mit ähnlichen Beschwerden vorgestellt, damals wurde ebenfalls die Verdachtsdiagnose eines Sweet-Syndroms gestellt.

Befunde. Hautbefund: Am rechten Unterschenkel bestand auf dem Boden einer erythematösen, randständig auch lividen, $7 \times 8 \mathrm{~cm}$ durchmessenden Plaque eine pralle ca. $4 \times 3 \mathrm{~cm}$ durchmessende Blase mit klarer Flüssigkeit. Am rechten Unterarm sowie am linken Unterschenkel zeigten sich multiple erythematöse sukkulente, zum Teil infiltrierte, druckdolente Papeln und Plaques sowie vereinzelt bräunliche, postinflammatorische Herde.

Histopathologie: In der Probebiopsie zeigte sich ein Ödem der papillären Dermis und ein dichtes diffuses granulozytäres Entzündungsinfiltrat.

Weitere Befunde: CRP 2,9 mg/dl. Blutsenkung $58 \mathrm{~mm} / \mathrm{h}$.

Diagnose. Sweet-Syndrom.

Therapie und Verlauf. Nach Biopsieentnahme und steriler Blasenpunktion am rechten Unterschenkel leiteten wir umgehend eine systemische steroidale Therapie ein mit Prednisolon $80 \mathrm{mg}$ p.o. in stufenweiser Dosisreduktion. Gleichzeitig wurde eine Therapie mit Dapson 100 mg pro Tag p.o. begonnen. Lokal behandelten wir an den Unterschenkeln zunächst mit antiseptischen Umschlägen im Wechsel mit lokalen Steroiden. Hierunter zeigte sich eine deutliche Befundbesserung.

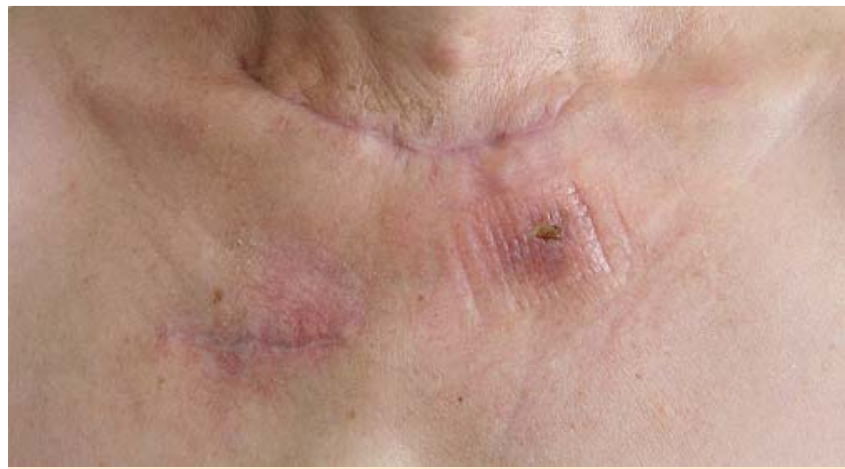

Abb. 3 Abzedierende neutrophile Dermatose bei einer 49-jährigen Patientin mit Paraproteinämie (Pat. 9).

\section{Fall 9}

Anamnese. Eine 49-jährige Patientin stellte sich stationär in der HNO-Klinik vor mit rezidivierenden Abzessen im Hals und Brustbereich. Mehrfache Inzisionen ergaben keinen Keimnachweis. Systemisch durchgeführte Antibiosen zeigten kein Ansprechen.

Befunde. Es fanden sich multiple schmerzhafte abzedierende polsterartige Hautveränderungen im Brust- und Halsbereich, z.T. mit erythematöser Mitbeteiligung der Kutis ( $\bullet$ Abb. 3). Erhöhte Temperatur bestand nicht, jedoch ausgeprägtes Krankheitsgefühl. In den Laboruntersuchungen zeigte sich ein erhöhtes CRP und eine Paraproteinämie. Im Rahmen der Tumorsuche konnte kein sicherer Nachweis einer myeloproliferativen Erkrankung festgestellt werden.

Diagnose. Abzedierende neutrophile Dermatose.

Therapie. Es erfolgte die Inzision eines sehr schmerzhaften Abzesses im Brustbereich und Gabe von $2 \mathrm{mg} / \mathrm{kg} / \mathrm{KG}$ Methylprednisolon p. o. für eine Woche, dann schrittweise Dosisreduktion. Bereits nach zwei Tagen zeigte sich eine deutliche Besserung.

\section{Diskussion}

$\nabla$

Der Begriff Pyoderma gangraenosum wurde von Busting 1930 erstmalig eingeführt [5] und die enge Beziehung der dramatisch zerstörenden Hauterkrankung mit entzündlichen Darmerkrankungen hergestellt. Eine weitere wichtige Beobachtung war das Pathergiephänomen beim Pyoderma grangraenosum und daher der Rat bei aktiven Ulzera von chirurgischen Exzisionen abzusehen. Häufig waren Minimaltraumata den ulzerierten Hautveränderungen vorausgegangen. Auch bei drei hier beschriebenen Patienten (Pat. 3, 4, 5) ( Tab. 2) wurden anamnestisch Minimaltraumata angegeben. Das Pathergiephänomen kann man auch gut an den Stellen verfolgen, wo eine histologische Sicherung durch eine Probeexzision (PE) vorgenommen wurde. Bei Pat. 1 und Pat. 2 vergrößerten sich die PE-Stellen rasch nach der Entnahme ( Tab. 2).

Seit der Erstbeschreibung sind neben den entzündlichen Darmerkrankungen eine Reihe von weiteren assoziierten Systemerkrankungen und Bedingungen beschrieben worden, wie die rheumatoide Arthritis, der Lupus erythematodes, die Psoriasis arthritis, Lebererkrankungen, Tumorerkrankungen und Medikamente [6,7]. Interessant ist, dass gerade die vegetierenden Varianten des Pyoderma gangraensoum seltener eine Assoziation mit entzündlichen Systemerkrankungen aufweisen [8-10]. Diese Variante ist insgesamt limitiert in der Ausprägung und Progression, häufig mit chronischem Verlauf von oberflächlichen 
Tab. 2 Klinische Daten der behandelten Patienten mit neutrophilen Dermatosen.

\begin{tabular}{|c|c|c|c|c|c|c|c|c|}
\hline $\begin{array}{l}\text { Pat. } \\
\text { Nr. }\end{array}$ & $\begin{array}{l}\text { Alter, } \\
\text { Geschlecht }\end{array}$ & Diagnose & $\begin{array}{l}\text { Initiale } \\
\text { Läsion }\end{array}$ & Lokalisation & $\begin{array}{l}\text { Assoziierte } \\
\text { Grunderkran- } \\
\text { kung }\end{array}$ & $\begin{array}{l}\text { Stationäre } \\
\text { Dauer (d) }\end{array}$ & Therapie & $\begin{array}{l}\text { Therapie- } \\
\text { ansprechen }\end{array}$ \\
\hline 1 & W, 18 & $\begin{array}{l}\text { Pyoderma } \\
\text { gangraenosum }\end{array}$ & Pustel & $\begin{array}{l}\text { gesamtes } \\
\text { Integument }\end{array}$ & Colitis ulcerosa & 25 & $\mathrm{Pr}, \mathrm{CyA}$ & PR \\
\hline 2 & $W, 40$ & $\begin{array}{l}\text { Pyoderma } \\
\text { gangraenosum }\end{array}$ & Makula & $\begin{array}{l}\text { Oberschenkel, } \\
\text { Unterschenkel, } \\
\text { Rücken }\end{array}$ & Sarkoidose & 30 & $\begin{array}{l}\mathrm{Pr}, \mathrm{CyA}, \\
\text { Infliximab }\end{array}$ & CR \\
\hline 3 & $\mathrm{M}, 70$ & $\begin{array}{l}\text { Pyoderma } \\
\text { gangraenosum }\end{array}$ & Trauma & $\begin{array}{l}\text { Unterschenkel } \\
\text { links }\end{array}$ & - & 104 & $\mathrm{Pr}, \mathrm{CyA}$ & CR \\
\hline 4 & W, 35 & $\begin{array}{l}\text { Pyoderma } \\
\text { gangraenosum }\end{array}$ & Makula & $\begin{array}{l}\text { Unterschenkel } \\
\text { beidseits }\end{array}$ & Hepatitis C & 74 & $\mathrm{Pr}, \mathrm{Az}$ & $C R$ \\
\hline 5 & W, 66 & $\begin{array}{l}\text { Pyoderma } \\
\text { gangraenosum }\end{array}$ & Makula & $\begin{array}{l}\text { Unterschenkel } \\
\text { beidseits }\end{array}$ & - & 62 & $\operatorname{Pr}, A z$ & PR \\
\hline 6 & W, 62 & Sweet-Syndrom & Makula & $\begin{array}{l}\text { Stamm, } \\
\text { Extremitäten }\end{array}$ & $\begin{array}{l}\text { Infekt der oberen } \\
\text { Atemwege }\end{array}$ & 7 & $\mathrm{Pr}$ & $C R$ \\
\hline 7 & W, 59 & Sweet-Syndrom & Knoten & $\begin{array}{l}\text { gesamtes } \\
\text { Ingument }\end{array}$ & $\begin{array}{l}\text { Infekt der oberen } \\
\text { Atemwege }\end{array}$ & 10 & $\mathrm{Pr}$ & CR \\
\hline 8 & W, 54 & Sweet-Syndrom & Makula & Extremitäten & - & 7 & $\mathrm{Pr}, \mathrm{Da}$ & CR \\
\hline 9 & W, 49 & $\begin{array}{l}\text { Abzedierende } \\
\text { neutrophilie } \\
\text { Dermatose }\end{array}$ & Knoten & Brust, Hals & Paraproteinämie & 10 & $\operatorname{Pr}$ & $C R$ \\
\hline
\end{tabular}

Ulzerationen. Die Diagnose ist oft schwieriger zu stellen, da im Gegensatz zum klassischen Pyoderma die Ulzera nicht unterminierend sind, weniger Gefäßverschlüsse in Randbereich zeigen und damit weniger stark livide im Ulkusrand imponieren ( $\bullet$ Abb. 2). Insgesamt sind die klinischen und histologischen Symptome weniger von Akuität geprägt. Die histologischen Veränderungen zeigen neben einem dermalen Infiltrat aus neutrophilen Granulozyten auch Lymphozyten, Plasmazellen und Fremdkörperriesenzellen. Daher wird im Schrifttum auch der angloamerikanische Begriff „superficial granulomatous pyoderma“ verwendet [10]. Die feingeweblichen Untersuchungen sind weder bei den klassischen noch bei den vegetierenden Formen spezifisch diagnostisch. Das klinische Krankheitsbild sowie der Verlauf sind führend bei der differenzialdiagnostischen Einordung. Ein weiterer typischer Vertreter der neutrophilen Dermatosen ist das Sweet-Syndrom, welches sich durch einen plötzlichen Beginn mit Fieber, Leukozytose und erythematösen z.T. sukkulenten Plaques auszeichnet ( Tab. 3) [11].

In mehr als 50\% der Fälle beobachtet man das Sweet-Syndrom in Assoziation mit anderen Erkrankungen oder Bedingungen (Medikamente, Schwangerschaft) (siehe Tab. 1) [12-14]. Von der Erkrankung sind Frauen häufiger betroffen (w:m, 4:1), wie auch in der hier vorgestellten Fallserie. Bei Pat. 6 und 7 sind vermutlich Streptokokkeninfekte der oberen Atemwege mit der Ausbildung des Sweet-Syndroms assoziiert. Histologisch findet sich beim Sweet-Syndrom meist eine eindrucksvolle dichte Infiltration von neutrophilen Granulozyten und nur wenigen Lymphozyten, Histiozyten und eosinophilen Granulozyten. Neutrophile Granulozyten können transepidermal migrieren und dabei subkorneale Pusteln ausbilden. Aber auch die Subkutis kann mit meist septalen entzündlichen Infiltraten mitbetroffen sein.

Die subkorneale Pustel ist die Leiteffloreszenz einer weiteren neutrophilen Dermatose, der subkornealen Pustulose (SneddonWilkinson) [15]. Klinisch finden sich zunächst kleine diskrete Pusteln, welche dann in weiteren Verlauf zu konfluierenden zirzinären z.T. bizarren pustulösen Formationen werden können. Nach einigen Tagen trocknen dann die Pusteln ein und heilen
Tab. 3 Diagostische Kriterien des Sweet-Syndroms.

Hauptkriterien
Plötzlicher Beginn mit typischen ödematösen, sukkulenten
erythematösen Plaques
Histologische Zeichen mit massiver Infiltration von neutrophilen
Granulozyten in der mittleren und oberen Dermis, häufig mit sub-
epidermalen Ödem
Nebenkriterien
Fieber oder Infektion
Begleitendes Fieber, Arthralgie, Konjunktivitis oder maligne Erkrankung
Leukozytose
Gutes Ansprechen auf systemische Kortikoide und kein Ansprechen
auf Antibiotika

mit Krusten und Schuppenbildung sowie typischen bräunlichen Hyperpigmentierungen ab. Episodische Verläufe sind nicht selten. In einigen Fällen gehen die akuten Hautveränderungen auch mit einem Pyoderma gangraenosum, entzündlichen Darmerkrankungen und IgA-Gammopathie einher [16,17].

Seltener als die subkorneale Pustulose sind die ekkrine Hidradenitis und die rheumatoide neutrophile Dermatose. Die ekkrine Hidradenitis wurde erstmalig von Harrist et al. bei Patienten mit akuter myeloischer Leukämie unter Polychemotherapien beschrieben [18]. Da die schmerzhaften, erythematösen, plaqueartigen Hautveränderungen immer wieder bei erneuten Chemotherapiezyklen auftraten, wurden als Ursachen eher die Chemotherapeutika angesehen als die zugrunde liegenden hämatologischen Erkrankungen ( $\bullet$ Tab. 4).

Zahlreiche nachfolgende Kasuistiken bestätigen diese Befunde. Am häufigsten sind die Extremitäten betroffen. Die Erkrankung ist selbstlimitierend, daher ist eine Therapie häufig nicht nötig. Bei jugendlichen Patienten fand sich die ekkrine neutrophile Hidradenitis auch ohne Grunderkrankung, meist an den Fußsohlen. In den feingeweblichen Untersuchungen fanden sich dichte Infiltrate von neutrophilen Granulozyten entlang der ekkrinen Schweißdrüsengänge. Ob die ekkrinen Schweißdrüsen als ein weiteres Ausscheidungsorgan des Körpers bei bestimmten Che- 
Tab. 4 Medikamentöse Auslöser.

\section{Medikamentöse Ursachen bei Sweet-Syndrom}

Lithium, Furosemid, Hydralazin, Minozyklin, Imatinib, Bortezomib, Trimethprim-Sulfamethoxazol, orale Kontrazeptiva

Medikamentöse Ursachen bei neutrophiler ekkriner Hidradenitis Bleomycin, Chlorambucil, Cyclophosamid, Cytarabin, Doxorubicin, Lomustin, Mitoxantrone, Topotecan, Vincristin

motherapeutika diese pathologische Entzündungsreaktion auslösen, ist mehrfach vermutet, aber bisher nicht endgültig bewiesen worden.

Die rheumatoide neutrophile Dermatose wurde von Ackerman 1978 erstmalig beschrieben [19]. Klinisch finden sich scharf begrenzte erythematöse bis violette Papeln und Plaques meist symmetrisch über den Gelenken und an den Streckseiten der Extremitäten. Im Gegensatz zu den bisher beschriebenen neutrophilen Dermatosen gehen diese Hautveränderungen nur mit der rheumatoiden Arthritis einher und meist parallel zu akuten Symptomen einer Gelenkentzündung. Histologisch findet sich ein dichtes Infiltrat aus neutrophilen Granulozyten im Bereich der gesamten Dermis. Die Erkrankung ist selbstlimitierend und dauert nur einige Tage an. Eine spezifische Therapie ist in den meisten Fällen nicht nötig $[20,21]$.

Die neutrophilen Dermatosen sind keine streng umrissene Gruppe von Erkrankungen, die einer festen Zuordnung unterliegen. Im weiteren Sinne können auch Entitäten wie die Pustulosen, das Erythema elevatum diutium, das Reiter-Syndrom, der Morbus Behçet und das SAPO-Syndrom genannt werden. Eine weitere sehr seltene reaktive neutrophile Dermatose ist die abzedierende neutrophile Dermatose [22,23]. Definiert sind aseptische Abzesse als tiefe sterile, meist runde Formationen, bestehend aus neutrophilen Granulozyten, die auf Antibiotika nicht ansprechen, sich aber nach Gabe von Kortikosteroiden dramatisch verbessern. Horiguchi u. Mitarb. beschrieben drei Fälle, zwei mit myelodysplastischem Syndrom und einen mit IgA-Myelom [23]. Auch die hier beschriebene Patientin hatte eine Paraproteinämie ohne sicheren Nachweis eines Myeloms oder Plasmozytoms. Innerhalb von wenigen Tagen bildeten sich neue Abzesse. Nach Gabe von Kortikosteroiden kam es auch in diesem Fall zu einer schnellen Besserung.

Die Pathogenese der neutrophilen Dermatosen ist noch nicht klar erforscht, jedoch ist es naheliegend, dass das pathogenetische Reaktionsmuster ähnlich ist oder sogar ein kontinuierliches Spektrum darstellt. Neben den meist gut wirksamen Kortikosteroiden stellt die anti-TNF $\alpha$-Antikörpertherapie eine gute Ergänzung des therapeutischen Spektrums dar, die in ihrer Wirksamkeit in den bisher dokumentierten Kasuistiken eindrucksvoll ist ( $\bullet$ Abb. 1). Multizenterstudien sind hier nötig, um diese Befunde bei diesen seltenen Entitäten zu überprüfen.

\section{Abstract}

\section{Reactive Neutrophilic Dermatosis \\ $\nabla$}

Reactive neutrophilic dermatoses like pyoderma gangrenosum or Sweet's syndrome describe distinct entities associated by similar pathologic characteristics. Histologic examination reveals a dense epidermal and/or dermal inflammatory infiltrate compos- ed of neutrophils. Typically, there is also an increased amount of neutrophiles in the peripheral blood and sometimes association with systemic inflammatory diseases. Common associated conditions are chronic inflammatory bowel disease, autoimmune and collagen vascular diseases and hematologic malignancies. Time of manifestation is often associated with exacerbation of the underlying disease. The exact pathogenesis of reactive neutrophilic dermatoses is still unknown, but these disorders typically respond to antiinflammatory therapies, especially to anti-TNF- $\alpha$ based therapeutic approaches.

\section{Literatur}

1 Callen JP. Neutrophilic dermatoses. Dermatol Clin 2002; 20: 409-419

2 Moschella SL. Review of so-called aseptic neutrophilic dermatoses. Australas J Dermatol 1983; 24: 55-62

3 Reuss-Borst MA, Pawelec G, Saal JG et al. Sweet's syndrome associated with myelodysplasia: possible role of cytokines in the pathogenesis of the disease. Br J Haematol 1993; 84: 356 - 358

4 Reuss-Borst MA, Müller CA, Waller HD. The possible role of G-CSF in the pathogenesis of Sweet's syndrome. Leuk Lymphoma 1994; 15: 261 264

5 Brunsting LA, Goeckerman WH, O'Leary PA. Pyoderma grangrenosum. Clinical and experimental observation in five cases occuring in adults. Arch Dermatol 1930; 22: 655-680

6 Powell FC, Schroeter AL, Su WP, Perry HO. Pyoderma gangrenosum: a review of 86 patients. QJ Med 1985; 55: $173-186$

7 Callen JP. Pyoderma gangrenosum and related disorders. Med Clin North Am 1989; 73: 1247-1261

8 Wildfeuer T, Albrecht G. Multilokuläres vegetierendes Pyoderma gangraenosum. Hautarzt 1999; 50: 217-220

9 Kim JW, Park JH, Lee D et al. Vegetative pyoderma gangrenosum in Behçet's disease. Acta Derm Venereol 2007; 87: 365 - 367

10 Del Cerro Heredero M, Sánchez Yus E, Gómez-Calcerrada MR et al. Superficial granulomatous pyoderma. Dermatology 1998; 196: 358 - 360

11 Sweet $R D$. An acute febrile neutrophilic dermatoses. Br J Dermatol 1964; 76: 349-356

12 Kemmett D, Hunter JA. Sweet's syndrome: a clinicopathologic review of twenty-nine cases. J Am Acad Dermatol 1990; 23: 503 - 507

13 Von den Driesch P. Sweet's syndrome (acute febrile neutrophilic dermatosis). J Am Acad Dermatol 1994; 31: 535-556

14 Cohen RP, Talpez M, Kurzrock R. Malignancy associated Sweet's syndrome. Review of world literature. J Clin Oncol 1988; 6: 1887

15 Sneddon IB, Wilkinson DS. Subcorneal pustular dermatosis. Br J Dermatol 1979; 100: 61-68

16 Kasha EE Jr, Epinette WW. Subcorneal pustular dermatosis (SneddonWilkinson disease) in association with a monoclonal IgA gammopathy: a report and review of the literature. J Am Acad Dermatol 1988; 19: $854-858$

17 Kohl PK, Hartschuh W, Tilgen W, Frosch PJ. Pyoderma gangrenosum followed by subcorneal pustular dermatosis in a patient with IgA paraproteinemia. J Am Acad Dermatol 1991; 24: 325 - 328

18 Harrist TJ, Fine JD, Berman RS et al. Neutrophilic eccrine hidradenitis. A distinctive type of neutrophilic dermatosis associated with myelogenous leukemia and chemotherapy. Arch Dermatol 1982; 118: $263-$ 266

19 Ackerman $A B$. Histologic diagnosis of inflammatory skin diseases. Philadelphia: Lea \& Febiger, 1978: 449-451

20 Scherbenske JM, Benson PM, Lupton GP, Samlaska CP. Rheumatoid neutrophilic dermatitis. Arch Dermatol 1989; 125: 1105-1108

21 Mashek HA, Pham CT, Helm TN, Klaus M. Rheumatoid neutrophilic dermatitis. Arch Dermatol 1997; 133: 757-760

22 Carvalho P, Cordel N, Courville $P$ et al. Cutaneous aseptic abscesses, manifestation of neutrophilic diseases. Ann Dermatol Venereol 2001; 128: $641-643$

23 Horiguchi Y, Lee SG, Matsumoto I et al. Abscess-forming neutrophilic dermatosis: report of three cases associated with hemopathies. Dermatology 1998; 197: 174-177 